\title{
Aftereffect of adaptation to uni-directional frequency change: Evidence for selective processing mechanism
}

\author{
Takuro Kayahara
}

Gifu MVL Research Center, Telecommunications Advancement Organization of Japan (TAO)/Intelligent Modeling Laboratory (IML), University of Tokyo,

2-11-16, Yayoi, Bunkyo-ku, Tokyo, 113-8656 Japan

E-mail: kayahara@iml.u-tokyo.ac.jp

( Received 17 July 2000, Accepted for publication 14 August 2000 )

Keywords: Uni-directional frequency change, Negative aftereffect, Direction selectivity, Frequency selectivity, Interaural transfer PACS number: 43.66. $\mathrm{Hg}, 43.66 . \mathrm{Mk}$

\section{Introduction}

This article describes the aftereffect of adaptation to a unidirectional frequency change (linear frequency glide) to investigate the characteristics of the selective mechanism for processing frequency change in the auditory system. After repeated exposure (adaptation) to linear frequency glides in a constant direction of change in frequency, subjects perceived fixed-frequency sinusoids as a frequency glide in the opposite direction.

Psychophysical evidence for the selective mechanism has come primarily from studies of adaptation to a periodic frequency-modulated tone [1]. However, it appears to be relevant to use a uni-directional change in frequency for adaptation to investigate the properties of the mechanism, because detailed characteristics of the mechanism, especially ones concerned with direction, can only be investigated with a uni-directional frequency change.

The selectivity of the mechanism to the direction of frequency change was argued by measuring the effect of adaptation to linear frequency glides on the detection threshold for uni-directional frequency change [2]. However, a question concerning the interpretation of adaptation was raised [3], and since then little effort has been made to investigate the mechanism for a uni-directional frequency change with the adaptation paradigm.

The purpose of this study was to confirm the existence of the mechanism for frequency changes in the human auditory system and clarify its selectivities to several attributes of unidirectional frequency change with a reevaluation of the adaptation paradigm.

\section{General Method}

The double staircase method, which consists of two series of staircases, was used to measure the magnitude of the aftereffect. These two series were randomly presented in every trial to minimize the effect of prediction by the subject. In each trial, following 10-times exposures to the adapting tone with an inter-stimulus interval of $100 \mathrm{~ms}$, the subject was exposed to a test tone and asked to judge the direction of the change in pitch as 'upward', 'downward', 'no change'. Sessions were interrupted when the subject's judgment reversed six times in each series of staircase.
For all experiments, the adapting tone was a linear frequency glide with a fixed frequency excursion of $80 \mathrm{~Hz}$ centered at $1,000 \mathrm{~Hz}$. The test tone was also a linear frequency glide with a fixed initial-point frequency throughout a session, and its end-point frequency was adaptively varied in each trial.

The average of the last three end-point frequencies at which a subject's judgment reversed in each series of staircases was used as a measurement of the session. The difference between the measurement of adaptation with a linear frequency glide and that with fixed-frequency sinusoids (control experiment) was taken as the magnitude of the aftereffect. What these procedures measured was the frequency excursion of the test tone necessary to "cancel" the effect of the adaptation.

The stimuli were digitally generated on a computer (Macintosh PowerMac 7300). The sampling rate was $44.1 \mathrm{kHz}$ with 16-bit quantization. The stimuli were converted to analog signals using a sound card (Emagic audiowerk8), and presented through a headphone (Sennheiser HDA200) to the subject's left ear in a sound-insulated room.

Three subjects with normal hearing participated in all experiments. They practiced until their performance appeared to be stable.

\section{Experiment 1}

\subsection{Method}

The magnitude of the aftereffect was measured for different directions of the adapting tone, either upward or downward. The duration of a single adapting tone was 250, 500, $750,1,000$, or $1,250 \mathrm{~ms}$. The frequency of the initial point of the test tone was fixed at $1,000 \mathrm{~Hz}$ and its duration was fixed at $750 \mathrm{~ms}$ throughout the experiment.

\subsection{Results}

Figure 1 shows the averaged magnitude of the aftereffect as a function of the duration of a single adapting tone for each direction of change of the adapting tone. For an upward change, test tones with some frequency excursion in the upward direction were judged to be stationary in pitch and correspondingly a stationary tone was perceived as changing downward. For a downward adapting tone, the reverse was true. These results indicate that the negative aftereffect occurred for both upward and downward adapting stimuli. 


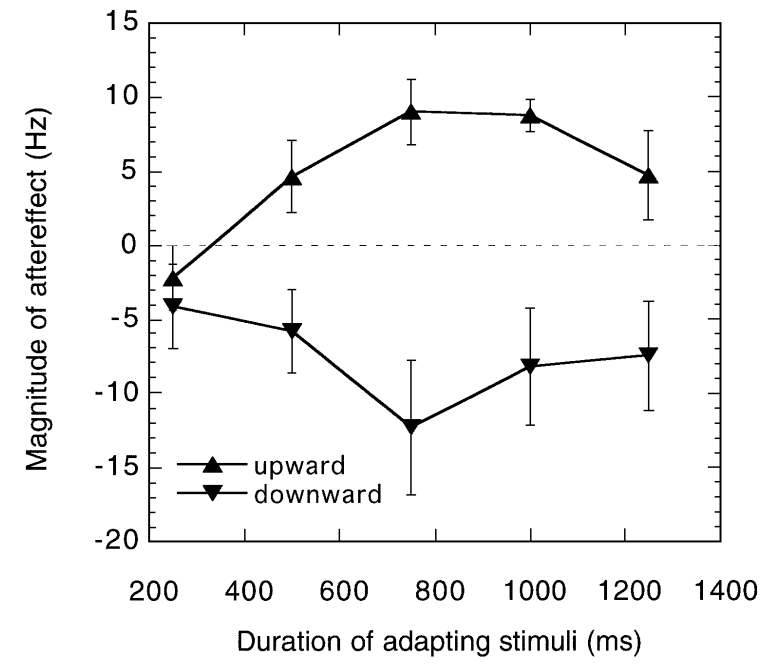

Fig. 1 Averaged magnitude of the aftereffect in experiment 1 . The sign of ordinate indicates the direction of excursion of the test tone ( + : upward, - : downward). Abscissa represents the duration of the adapting tone. Legend indicates the direction of the adapting tone. Error bars indicate the standard deviation across subjects.

The magnitude of the aftereffect was largest when the duration of the single adapting tone was $750 \mathrm{~ms}$. This peak of the effect can be interpreted as the selectivity to the rate of frequency change since the excursion of the adapting stimuli was fixed throughout the experiment.

\section{Experiment 2}

\subsection{Method}

The magnitude of the aftereffect was measured for several durations of test tone to confirm the rate selectivity of the aftereffect suggested in experiment 1 . The adapting tone was the same as in experiment 1 except that only the upward direction was used in this experiment. The duration of the test tone was 250,500 , or $750 \mathrm{~ms}$. The frequency at the initial point of the test tone was fixed at $1,000 \mathrm{~Hz}$.

\subsection{Results}

Figure 2 shows the averaged magnitude of the aftereffect as a function of the duration of a single adapting tone for each test tone duration. In almost every condition, test tones with upward excursions were judged as stationary, indicating that a negative aftereffect occurred. For all test tone durations, the largest aftereffect was obtained not at one particular duration of the single adapting tone (particular rate of change) but at the duration equal to that of the test tone.

\section{Experiment 3}

\subsection{Method}

The effect of the difference between center frequency of the adapting tone and the initial-point frequency of the test tone was measured to investigate the frequency selectivity of the mechanism. The duration of the single adapting tone was fixed at $750 \mathrm{~ms}$ with the direction fixed to upward. The frequency of the initial point of the test tone was 800, 900, 1,000, 1,100 , or $1,200 \mathrm{~Hz}$. Its duration was fixed at $750 \mathrm{~ms}$.

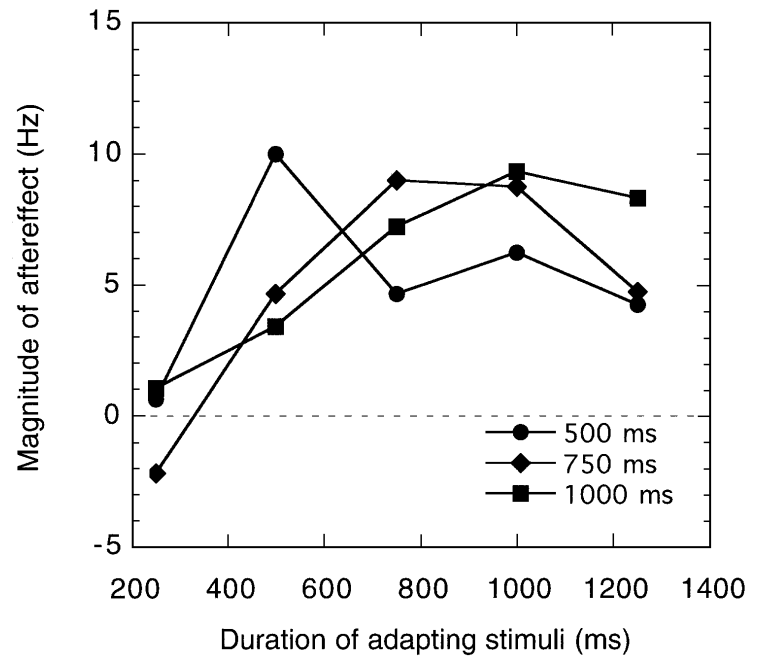

Fig. 2 Averaged magnitude of the aftereffect in experiment 2. The sign of ordinate indicates the direction of excursion of the test tone ( + : upward, - : downward). Abscissa represents the duration of the adapting tone. Legend indicates the duration of the test tone.

\subsection{Results}

Figure 3 shows the averaged magnitude of the aftereffect as a function of the initial-point frequency of the test tone. When the initial-point frequency of the test tone was identical to the center frequency of the adapting tone $(1,000 \mathrm{~Hz})$, a negative aftereffect occurred. However, when initial-point frequency of the test tone was about $100 \mathrm{~Hz}$ away from the center frequency of the adapting tone, no aftereffect was obtained.

\section{Experiment 4}

\subsection{Method}

The magnitude of the aftereffect was measured when the adapting tone was presented to one ear and the test tone was presented to the other ear to investigate interaural transfer of the aftereffect. The duration of the single adapting tone was 500,750 , or $1,000 \mathrm{~ms}$ with the direction fixed to upward. The duration of the test tone was identical to that of the single adapting tone, providing the most effective combinations of duration indicated in experiment 2 . The adapting tone was presented to the left ear, and the test tone was presented to the right ear through headphones.

\subsection{Results}

Figure 4 shows the averaged magnitude of the aftereffect for two stimulus presentation conditions (contralateral or ipsilateral). The data obtained in experiment 2 was replotted as that of the ipsilateral condition. In the contralateral condition, the negative aftereffect was not obtained, indicating no interaural transfer of the aftereffect.

\section{Discussion}

The results of experiment 1 show that adaptation to a linear frequency glide caused a negative aftereffect whose direction was dependent on the direction of the adapting tone. These results suggest that there is a mechanism for processing frequency change and that it has selectivity to the direction of 
change. Directional selectivity of the mechanism was reported in previous psychophysical studies using adaptation [2], supporting the results obtained in this study. Neurophysiological studies have identified the neurons that selectively respond to a particular direction of frequency change in the auditory pathways of various animals $[4,5]$.

The results of experiment 2 show that the largest aftereffect was obtained when the duration of a single adapting tone was identical to that of the test tone. If the aftereffect occurred in a rate selective manner, then the largest aftereffect should be obtained at a particular duration of the single adapting tone regardless of the duration of the test tone, because the frequency extent of the adapting tone was fixed in this experiment. Therefore, these results suggest that the mechanism might not be rate selective. Instead, the mechanism might represent a change in frequency as the extent of change over the duration of stimulus.

The results of experiment 3 show that the magnitude of the aftereffect was dependent on the difference between the center frequency of the adapting tone and the initial-point frequency of the test tone. They suggest that the mechanism has frequency selectivity; that is, the mechanism might respond only to a changing frequency occurring within a limited extent of the frequency band.

The results of experiment 4 show that the aftereffect did not transfer to the contralateral ear from the adapted one. This lack of interaural transfer suggests that the mechanism might be located somewhere in the monaural pathways or in the peripheral parts of the auditory system.

\section{References}

[1] R. H. Kay and D. R. Matthews, "On the existence in human auditory pathways of channels selectivity tuned to the modulation present in frequency modulation tones," J. Physiol. (Lond.) 225, 657-667 (1972)

[2] R. B. Gardner and J. P. Wilson, "Evidence for direction-specific channels in the processing of frequency modulation," J. Acoust. Soc. Am. 66, 704-709 (1979).

[3] G. H. Wakefield and N. F. Viemeister, "Selective adaptation to linear frequency-modulated sweeps: Evidence for direction-specific FM channels?," J. Acoust. Soc. Am. 75, 1588-1592 (1984).

[4] R. Britt and A. Starr, "Synaptic events and discharge patterns of cochlear nucleus cells. II. Frequency-modulated tones," J. Neurophysiol. 39, 179-194 (1976).

[5] B. Tian and J. P. Rauschecker, "Processing of frequencymodulated sounds in the cat's anterior auditory field," J. Neurophysiol. 71, 1959-1975 (1994).

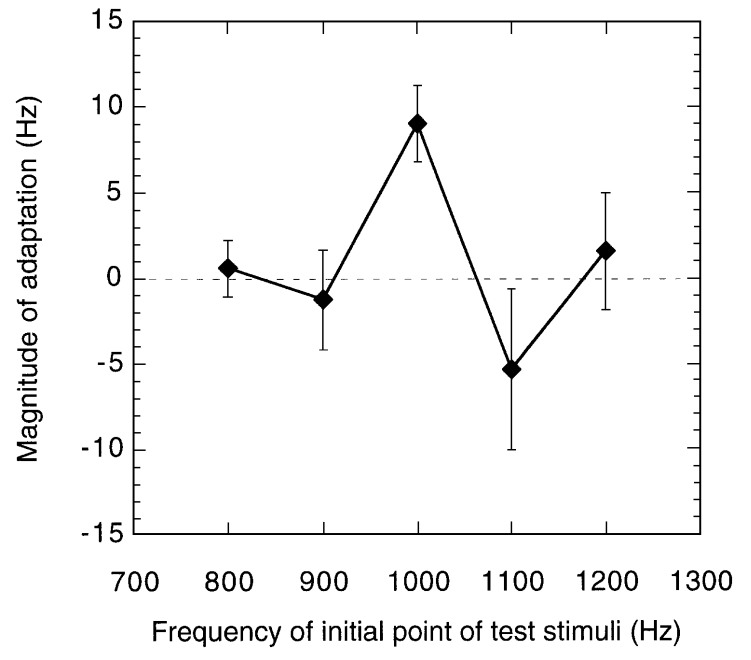

Fig. 3 Averaged magnitude of the aftereffect in experiment 3. The sign of ordinate indicates the direction of excursion of the test tone (+: upward, - : downward). Abscissa represents the initial-point frequency of the test tone. Error bars indicate the standard deviation across subjects.

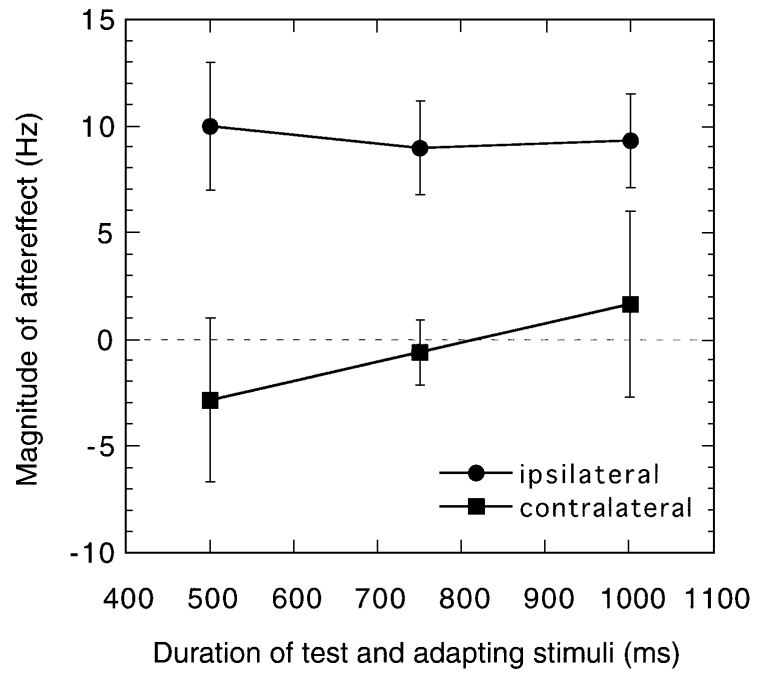

Fig. 4 Averaged magnitude of the aftereffect in experiment 4 . The sign of ordinate indicates the direction of excursion of the test tone ( + : upward, - : downward). Abscissa represents the duration of the adapting tone and test tone. Legend indicates the condition of stimulus presentation. Error bars indicate the standard deviation across subjects. 